\title{
OCT4B1 promotes cell growth, migration and invasion suppressing sensitivity to oxaliplatin in colon cancer
}

\author{
KUN-MING WEN ${ }^{1}$, GUI-HAI ZHANG ${ }^{2}$, JIANG LI ${ }^{1}$, ZHENG-QUAN CHEN ${ }^{1}$, \\ YI-LIN CHENG ${ }^{1}, \mathrm{XUAN} \mathrm{SU}^{1}$ and QING-LIANG ZENG ${ }^{1}$ \\ Departments of ${ }^{1}$ Gastrointestinal Surgery and ${ }^{2}$ Abdominal Oncology, The Affiliated Hospital \\ of Zunyi Medical College, Zunyi, Guizhou 563000, P.R. China \\ Received April 15, 2015; Accepted July 16, 2015
}

DOI: $10.3892 /$ or.2015.4286

\begin{abstract}
OCT4B1, a splice variant of OCT4, is a key regulator in maintaining the properties of pluripotency and self-renewal in embryonic stem (ES) cells. Recent results have shown that OCT4B1 is involved in tumorigenesis. However, the contribution of OCT4B1 in the tumorigenesis and drug resistance of colon cancer remains to be determined. The aim of the present study was to determine whether OCT4B1, which maintains the stemness of ES cells, promoted cell growth by facilitating transition of the cell cycle and reduced apoptosis in colon cancer and drug-resistant cells using flow cytometry and western blotting. The results showed that, OCT4B1 promoted the growth of colon cancer and drug-resistant cancer cells by maintaining the activity of ES cells and by facilitating the transition of the cell cycle and reducing apoptosis. Additionally, OCT4B1 was able to reduce sensitivity to oxaliplatin by altering the expression of two important mediators in drug resistance, $\mathrm{P}-\mathrm{gp}$ and ABCG2 [ATP-binding cassette, sub-family G (WHITE), member 2]. Furthermore, OCT4B1 enhanced the ability of migration and invasion through alteration of the epithelial-to-mesenchymal transition (EMT) in colon cancer. In conclusion, to the best of our knowledge, the results demonstrated for the first time that OCT4B1 functions as an oncogene in colon cancer and provides the development of novel therapeutic strategies to treat colon cancer, particularly drug resistance.
\end{abstract}

\section{Introduction}

Cancer is the second cause of mortality, with colon cancer being the second most lethal cause of cancer in women and the third in men, worldwide (1). Approximately 102,900 cases

Correspondence to: Dr Kun-Ming Wen, Department of Gastrointestinal Surgery, The Affiliated Hospital of Zunyi Medical College, 149 Da-Lian Road, Zunyi, Guizhou 563000, P.R. China E-mail: kunmingwen@126.com

Key words: OCT4, drug resistance, tumorigenesis, colon cancer, EMT, apoptosis, cell cycle were diagnosed with and 51,370 patients succumbed to causes associated with colon and rectal cancer combined in the United States in 2010 (2). Previous findings showed that colon cancer is a multifactorial and multistep disease and is associated with the mutations of many specific genes and the alterations of epigenetic and non-genotoxic factors (3). Thus, gaining knowledge on the specific molecular markers is crucial for the diagnosis and treatment of this disease. One of the factors involved in understanding the mechanism of tumor is the activity of oncogenes or tumor suppressors (4). Alteration of oncogenes or tumor-suppressor activity often occurs at the critical points of the development of colon cancer (4). For example, cytokines from the TNF family have potent inflammatory activities and play an important role in cancer development by regulating apoptosis in colon cancer (5). Chen et al reported that the tumor suppressor p53 promotes mitochondrial DNA base excision to regulate the development of colon cancer (6). However, the molecular mechanism of colon cancer development and progression remains unknown.

For patients, most early lesions are treated using surgery, radiation and chemotherapy. However, resistance to chemotherapy is the leading cause of treatment failure in colon cancer (7). The main reason for this failure is that cancer cells develop resistance to chemotherapy drugs. Thus, clarifying the potential mechanisms underlying drug resistance in cancer can improve the survival rate in patients. Depending on the type of drug resistance, there are different structures and mechanisms of action (8). The well-known ABC transporter family can partly explain the mechanisms of drug resistance (9). The ABC transporter family includes P-glycoprotein (P-gp, encoded by the $A B C B 1$ gene), MDR-associated protein 1 (MRP1, encoded by the $A B C C 1$ gene) and ABC subfamily $\mathrm{G}$ member 2 (ABCG2) (10). It has been shown that oncogenes or tumor suppressors, such as octamer-binding protein 4 (OCT4), which contribute to the tumorigenesis and progression of cancer, also regulate the chemosensitivity of tumor cells.

OCT4 is a POU homeodomain transcription factor and encodes three transcripts including a long main variant (OCT4A) and two alternatively spliced variants (OCT4B and OCT4B1) (11). The variants are associated with the pluripotency and self-renewal properties of embryonic stem (ES) cells $(7,12)$. Based on the pluripotency and self-renewal role in ES cells, OCT4 plays important roles in the tumorigenesis and 
progression of cancer. Li et al reported that OCT4 is capable of repressing $\mathrm{T} g f \beta 3$ and $\mathrm{T} g f \beta \mathrm{R} 3$ and downregulating the epithelial-to-mesenchymal transition (EMT) regulator Snail (13). In esophageal carcinoma, OCT4 regulates CCND1 expression to promote cell progression and accelerate cell proliferation and invasion (14). Atlasi et al reported that the long variant OCT4A was found to be required for the pluripotency properties of ES cells (11). OCT4B1, which lacks DNA-binding activities and is located in the cytoplasm, has also been suggested to have a vital catalytic role in pluripotency. Accumulating evidence suggests that OCT4B1 contributes to the initiation and progression of cancer (15). In bladder cancer, OCT4B1 was upregulated and promoted the tumorigenic process (15). Asadi et al and Mirzaei et al also found that OCT4B1 was highly expressed in gastric cancer and acts as an anti-apoptotic factor $(16,17)$. However, the function of OCT4B1 in colon cancer and drug-resistant cells remain to be determined.

In the present study, we investigated the role of OCT4B1 in the malignant phenotype of colon cancer and drug-resistant cancer cells. We found that OCT4B1 promoted the growth of colon cancer and drug-resistant cancer cells, by maintaining the activity of ES cells, and by facilitating transition of the cell cycle and reducing apoptosis. OCT4B1 reduced sensitivity to oxaliplatin by altering the expression of P-gp and ABCG2. In addition, the results revealed that, OCT4B1 contributes to the migration and invasion by altering EMT in colon cancer and drug-resistant cells. Taken together, the results indicate that OCT4B1 functions as an oncogene and a mediator of the drug resistance process in colon cancer.

\section{Materials and methods}

Cell culture, transfection and stable cell line selection. The human SW480 and SW620 colon cancer cell lines were cultured in Dulbecco's modified Eagle's medium (DMEM; Gibco, Gaithersburg, MD, USA), supplemented with $10 \%$ dialyzed fetal bovine serum (FBS) and 1\% PS (100 U/ml penicillin and $100 \mathrm{~g} / \mathrm{ml}$ streptomycin). The cell lines were incubated at $37^{\circ} \mathrm{C}$ in a humidified chamber supplemented with $5 \% \mathrm{CO}_{2}$. Transfection was performed with Lipofectamine 3000 reagent (Invitrogen Life Technologies, Carlsbad, CA, USA) according to the manufacturer's instructions.

Stably transfected cells were selected by adding G418 to the culture medium after transfection $48 \mathrm{~h}$ later. Individual colonies were selected 2 weeks after transfection, and OCT4B1 levels were quantified using western blot analysis.

Construction of vectors. The CDS of human OCT4B1 was amplified from human SW480 cDNA by PCR using the oligonucleotide primers: sense, 5'-CGCGGATCCCGCCACCAT GCACTTCTAC-3' and antisense, 5'-CCGGAATTCCTACTC CTCTTCATGGGTGAG-3'. PCR was performed by denaturing the DNA at $94^{\circ} \mathrm{C}$ for 4 min, followed by 30 cycles of amplification: $94^{\circ} \mathrm{C}$ for $45 \mathrm{sec}, 56^{\circ} \mathrm{C}$ for $40 \mathrm{sec}, 72^{\circ} \mathrm{C}$ for $1 \mathrm{~min}$, and a final extension step at $72^{\circ} \mathrm{C}$ for $10 \mathrm{~min}$. The product was $572 \mathrm{bp}$, and it was cloned into the pcDNA3.1 vector sites (EcoRI and Bam $\mathrm{HI})$. The resulting construct pcDNA3.1/OCT4B1 (OCT4B1) was confirmed by DNA sequencing.

The shRNA of OCT4B1 (shR-OCT4B1) was annealed and cloned into the pSilencer2.1 vector sites. A 70-bp double-strand fragment was obtained via an annealing reaction using the two single strands: ShR-OCT4B1-Top, 5'-GATCCCAG ACTACCCTCACCCATGTTCAAGAGACATGGGTGAGG GTAGTCTGTTTTTTGGAAA-3'; ShR-OCT4B1-Bot, 5'-AGC TTTTCCAAAAAACAGACTACCCTCACCCATGTCTCT TGAACATGGGTGAGGGTAGTCTGCG-3'. The negative control pSilencer2.1/negative control (shR-Ctrl) expressed a hairpin shRNA with limited homology to all known sequences in the human genome.

$R N A$ extraction and $R T-q P C R$. Total RNA was extracted from cells using TRIzol reagent (Invitrogen Life Technologies) according to the manufacturer's instructions. For RNA integrity assessment, part of an RNA sample was used for concentration and purity measurement (by A260 and A280 spectrophotometry) and another part of the sample was run on a $1.5 \%$ denaturing agarose gel stained with ethidium bromide. A ratio of the absorbance at 260 and $280 \mathrm{~nm}$ (A260/280) of 1.8-2 was accepted. Sharp, clear $28 \mathrm{~S}$ and $18 \mathrm{~S}$ rRNA bands at a 2:1 ratio (28S:18S) were good indicator that the RNA was completely intact.

Reverse transcriptase-quantitative PCR (RT-qPCR) was performed to detect the relative transcript levels of OCT4B1 according to the manufacturer's instructions and analyzed using the ABI 7300 RT-PCR system (Life Technologies, Carlsbad, CA, USA). Briefly, a cDNA library was generated using M-MLV reverse transcriptase (Promega, Madison, WI, USA) and oligo (dT) with $5 \mathrm{lg}$ of extracted RNA, and this cDNA was used for the amplification of OCT4B1 and $\beta$-actin. PCR was performed under the following conditions: $94^{\circ} \mathrm{C}$ for $4 \mathrm{~min}$ followed by 40 cycles of $94^{\circ} \mathrm{C}$ for $1 \mathrm{~min}, 56^{\circ} \mathrm{C}$ for $1 \mathrm{~min}$ and $72^{\circ} \mathrm{C}$ for $1 \mathrm{~min}$. The primers used were: OCT4B1 forward, 5'-TGAATCCCGAATGGAAAGG-3' and reverse, 5'-GGA ACCCACCAAATAGAAC-3'; and GAPDH forward, 5'-AAC GGATTTGGTCGTATTG-3' and reverse, 5'-GGAAGATGG TGATGGGATT-3'. The primers were produced by AuGAT Inc. (Beijing, China). The relative expression levels of the gene of interest were calculated using the $2^{-\Delta \Delta \mathrm{Ct}}$ method.

Cell growth and cytotoxicity assay. The SW480 and SW620 cells were seeded in 96-well plates at $1 \times 10^{3}-1 \times 10^{4}$ cells/ well. For cell cytotoxicity assays, 24,48 and $72 \mathrm{~h}$ after being seeded, the cells were incubated with $15 \mu \mathrm{l}$ of MTT (at a final concentration of $0.5 \mathrm{mg} / \mathrm{ml}$ ) at $37^{\circ} \mathrm{C}$ for another $4 \mathrm{~h}$. After incubation the medium was removed and the precipitated formazan was dissolved in $100 \mathrm{ml}$ of DMSO. Following agitation for $10 \mathrm{~min}$, the absorbance at $570 \mathrm{~nm}$ was detected using a Quant Universal microplate spectrophotometer (Bio-Tek Instruments, Winooski, VT, USA). Each experiment was repeated in triplicate.

In cytotoxicity assays, cells were seeded in a 96-well plate $\left(5 \times 10^{3}\right.$ cells/well). After $24 \mathrm{~h}$, the cell medium was replaced with fresh medium containing six different concentrations of oxaliplatin $(0,0.3125,0.625,1.25,2.5$ and $5 \mathrm{mg} / \mathrm{ml})$. The cells were then incubated at $37^{\circ} \mathrm{C}$ for $24 \mathrm{~h}$ and cell viability was determined by MTT assays. The absorbance at $570 \mathrm{~nm}$ (A570) of each well was read on a spectrophotometer. The inhibition rate (IR) was calculated according to the formula: $\mathrm{IR}=[(\mathrm{XY}) / \mathrm{X}] \times 100 \%$ from each group. The concentration at which each drug produced $50 \%$ inhibition of growth $\left(\mathrm{IC}_{50}\right)$ 
was estimated using the IR. Three independent experiments were performed in triplicate.

Cell cycle analysis and apoptosis by flow cytometry. Stably transfected cells were plated in duplicate in 6-well plates and incubated for $24 \mathrm{~h}$ in complete culture medium. One group of cells was deprived of serum for $24 \mathrm{~h}$ prior to being harvested, whereas another group of cells was returned to complete medium for another $24 \mathrm{~h}$ prior to being harvested. The cells were collected by centrifugation, fixed in $95 \%$ (v/v) ethanol and stored at $-20^{\circ} \mathrm{C}$ overnight. After washing with phosphate-buffered saline (PBS), the cells were resuspended in propidium iodide (PI) staining buffer (PBS, 0.1\% Triton X-100, $60 \mathrm{~g} / \mathrm{ml}$ PI, $0.1 \mathrm{mg} / \mathrm{ml}$ DNase-free RNase and $0.1 \%$ trisodium citrate) for $30 \mathrm{~min}$ on ice. The DNA content was analyzed using a FACSCalibur flow cytometer (BD Biosciences, San Jose, CA, USA) and Cell Quest software (BD Biosciences). For cell apoptosis, stably transfected cells were determined using a FITC-Annexin V/PI Apoptosis Detection kit (KeyGEN, Nanjing, China) by FCM (BD Biosciences). The percentage of apoptotic cells was analyzed by Cell Quest software.

Western blot analysis. Total cell extracts were extracted using radioimmunoprecipitation assay (RIPA) lysis buffer lysed at $4^{\circ} \mathrm{C}$ for $30 \mathrm{~min}$ and the proteins were harvested. The cell extracts were cleared by centrifugation at $12,000 \mathrm{x}$ g for $10 \mathrm{~min}$ at $4^{\circ} \mathrm{C}$, and the supernatant was used for western blot analysis. The protein concentration of the cell lysates was determined using BCA reagents from Promega. Approximately $25 \mu \mathrm{g} /$ lane of proteins was loaded onto a $10 \%$ SDS denaturing polyacrylamide gel, separated by electrophoresis and transferred to nitrocellulose membranes. The membranes were incubated overnight at $4^{\circ} \mathrm{C}$ with anti-OCT4B1, anti-E-cadherin, anti-N-cadherin, anti-vimentin, anti-CD44, anti-CD133, anti-P-gp, anti-ABCG2 and anti-GAPDH (Tianjin Saier Biotech, Tianjin, China). The membranes were washed and incubated with a horseradish peroxidase (HRP)-conjugated secondary antibody. Protein was visualized using enhanced chemiluminescence and exposing the membranes to autoradiographic film. LabWorks ${ }^{\mathrm{TM}}$ Image Acquisition and Analysis software (UVP) were used to quantify the band intensities.

In vitro invasion assays. In vitro cell invasion assays were performed using Transwell chambers (pore size of $8 \mu \mathrm{M}$; Costar, Corning, NY, USA) with $2 \mathrm{mg} / \mathrm{ml}$ Matrigel (Clontech, Mountain View, CA, USA) according to the manufacturer's instructions. Transfected cells were seeded in the upper chamber of the insert at a density of $1 \times 10^{5}$ cells (SW480 or SW620) and covered with $250 \mu 1$ of serum-free medium. For screening, any cells that had not migrated after $20 \mathrm{~h}$ were removed and the membranes were stained with a $2 \%$ crystal violet solution for $10 \mathrm{~min}$ and placed on a glass slide. The cells adhering to the lower membrane of the inserts were counted. Three different fields of images (magnification, x5) were taken for each membrane, and the number of migratory cells was counted. The mean of triplicate assays for each experimental condition was used.

In vitro wound-healing assay. A cell wound-healing assay was performed as described previously (19). Transfected cells were seeded into 24 -well plates coated with gelatin prior to wounding. Cell wound-healing assay was induced by adding medium supplemented with $10 \%$ FBS and $1 \%$ PS (100 U/ml penicillin and $100 \mathrm{~g} / \mathrm{ml}$ streptomycin). The wound was created by scraping a conventional pipette tip across the monolayer. It typically took 12 and $24 \mathrm{~h}$ for wound closure to occur in SW480 and SW620 cells. Images of three different fields were captured using NIS Elements F 2.20 imaging software (Nikon, Tokyo, Japan).

Statistical analysis. Data were expressed as means \pm SD. Statistical analyses were performed using a paired t-test to compare data. Statistical significance $(\mathrm{P}<0.05)$ was determined using a Student's t-test.

\section{Results}

Screening of OCT4B1 stably expressed cells. To examine the role of OCT4B1 on cell phenotypes in colon cancer, we first constructed an OCT4B1 overexpression construct (pcDNA3.1/OCT4B1, OCT4B1) and an OCT4B1 interference vector (pSilencer 2.1-U6/OCT4B1, shR-OCT4B1) to overexpress or knockdown OCT4B1. We then screened single-cell cloning cells with G418 to gain a stable expression of OCT4B1 in colon cancer cells. We obtained four stably expressed cells that were overexpressed by OCT4B1 (SW480/OCT4B1 and SW620/OCT4B1) and the negative control cells (SW480/pcDNA3.1 and SW620/pcDNA3.1) (Fig. 1A). The expression of OCT4B1 was assessed by western blot analysis in the stably expressed cells (Fig. 1B and C). As expected, in the OCT4B1 overexpression group the protein level of OCT4B1 was increased $21.05 \%$ (SW480/OCT4B1) and $40.04 \%$ (SW620/OCT4B1) compared with the control group (Fig. 1B and C). Concurrently, we obtained the OCT4B1 knockdown stably expressed cells in colon cancer (SW4801shROCT4B1) and their negative control cells (SW480/shR-Ctrl) (Fig. 1A). OCT4B1 expression decreased $\sim 30.41 \%$ compared with the negative control (Fig. $1 \mathrm{~B}$ and $\mathrm{C}$ ). To examine the role of OCT4B1 in the formation and development of drug resistance the oxaliplatin-resistant stably expressed cells (SW480/OXR-shR-OCT4B1 and SW620/OXR-shR-OCT4B1) and their negative control cells (SW480/OXR-shR-Ctrl and SW620/OXR-shR-Ctrl) were obtained (Fig. 1A). OCT4B1 expression decreased $\sim 31.44 \%$ (SW480/OXR-shR-OCT4B1) and 31.09\% (SW620/OXR-shR-OCT4B1) compared with the negative control (Fig. 1B and $\mathrm{C}$ ). Thus, we screened and identified the stably expressed cells that altered the OCT4B1 expression for further study.

OCT4B1 enhances cell growth by accelerating cell cycle progression and reducing apoptosis and maintaining EC cell property. Given that OCT4B1 acts as an oncogene in many types of cancer, we performed an MTT assay to examine the impact of OCT4B1 on the cell growth of colon cancer. OCT4B1 knockdown stably expressed cells were used. Knockdown of OCT4B1 caused a reduction of cell viability in SW480/shR-OCT4B1 compared with the negative control (Fig. 2A). In the drug-resistant cells, the knockdown of OCT4B1 (SW480/OXR-shR-OCT4B1 and SW620/OXR-shROCT4B1) also reduced cell viability. 
A
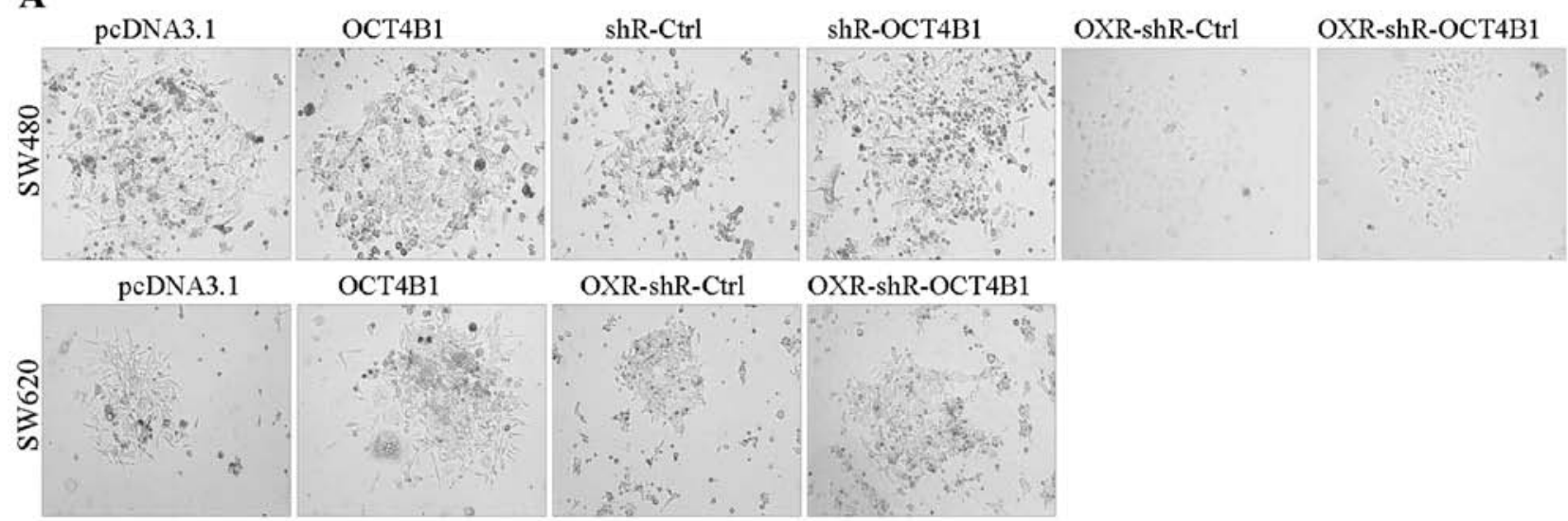

B
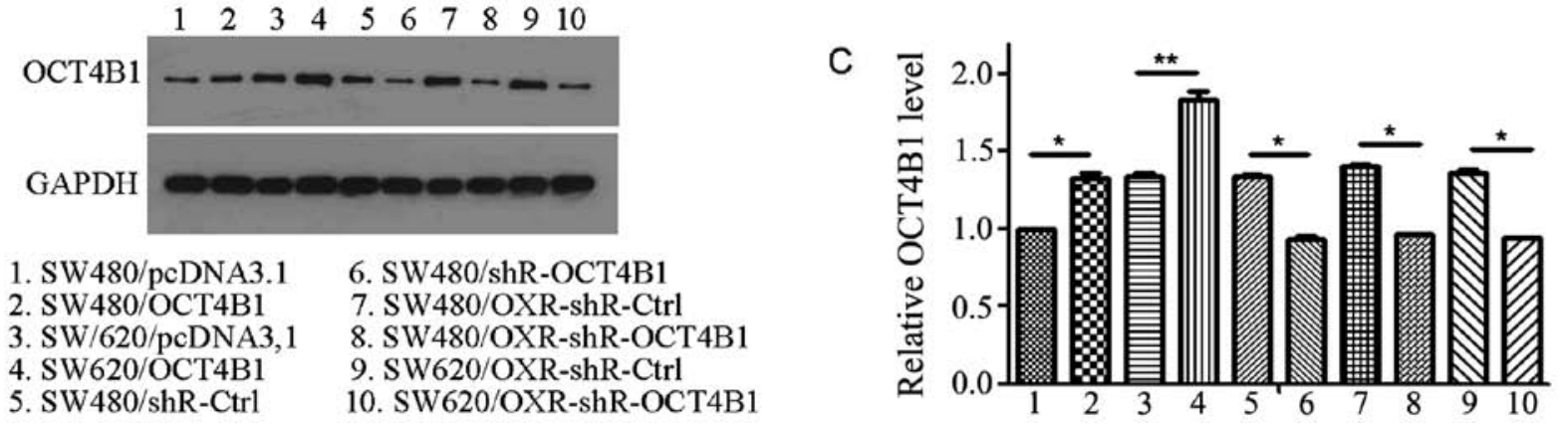

Figure 1. Screening of stably expressed cells. (A) Images of stably expressed cells overexpressed or knocked down by OCT4B1. SW480/OCT4B1 and SW480/pcDNA3.1 are the stably expressed cells that OCT4B1 overexpressed. SW480/shR-OCT4B1 and SW480/shR-Ctrl are shR-OCT4B1 stably expressed cells and the negative control in SW480 cells. SW480(620)/OXR-shR-OCT4B1 and SW480(620)/OXR-shR-Ctrl are the OCT4B1 stably expressed cells and the negative control in SW480(620) drug-resistant cells. (B) Western blot analysis was used to detect the expression of OCT4B1 in the stably expressed cells. GAPDH was used as the control. (C) The quantification of expression of OCT4B1 in western blot analysis. OCT4, octamer-binding protein 4 . ${ }^{*} \mathrm{P}<0.05$ and ${ }^{* *} \mathrm{P}<0.01$.

Mounting evidence indicates that cell cycle and apoptosis can partially explain the change of cell viability (18). To investigate the mechanisms underlying the regulation of cell viability, we examined the alteration in cell cycle progression caused by OCT4B1 in colon cancer stably expressed cells. Results of the flow cytometric analysis (FCM) showed that the overexpression of OCT4B1 in SW480 cells decreased the percentage of G1 phase from 72.40 to $62.47 \%$ and increased the percentage of cells in the S phase from 19.52 to $29.66 \%$ (Fig. 2B). Similarly, OCT4B1 overexpression led to an increment of $\mathrm{G} 1$ phase from 72.89 to $83.07 \%$, and a reduction of $\mathrm{S}$ phase from 19.08 to $13.01 \%$ in SW620 stably expressed cells (Fig. 2C). By contrast, the inhibition of OCT4B1 in SW480/shR-OCT4B1 cells increased the percentage of G1 phase and decreased the percentage of S phase (Fig. 2D). These results indicated that OCT4B1 contributes to cell growth by promoting the transition from $\mathrm{G} 1$ and $\mathrm{S}$ phases to G2 phase. Furthermore, we detected the effect of OCT4B1 on the drug-resistant cells. The percentage of G1-phase cells was increased from 66.85 to $77.41 \%$, whereas the percentage of S and G2 phase cells was decreased from 26.54 and $6.61 \%$ to 17.03 and $5.56 \%$ in SW480/shR-OCT4B1-OXR cells compared with the negative control (Fig. 2E). Similar results were obtained in the SW620/shR-OCT4B1-OXR stably expressed cells (Fig. 2F). Thus, these results suggesetd that OCT4B1 is capable of altering cell cycle progression in colon cancer, especially in drug-resistant cancer.
In parallel, the apoptosis index of OCT4B1 knockdown stably expressed cells was detected by FCM. The apoptosis index of SW480/shR-OCT4B1 cells was increased by 29 -fold compared with the control group (Fig. 3A). Similarly, the effect of OCT4B1 on the drug-resistant cells was identified. The apoptosis index of SW480/OXR-shR-OCT4B1 and SW620/OXR-shR-Ctrl stably expressed cells was increased by $\sim 18$ - and $\sim 16$-fold, respectively, compared with the negative control (Fig. 3A).

We also detected the effect of EC cell property. Two EC cell biomarkers, CD44 and CD133, were examined using western blot analysis. The expression of CD44 and CD133 was 1.40- and 6.29-fold in the SW480/OCT4B1 cells compared with the control group. Knockdown of OCT4B1 led to a 15.17 and $249.13 \%$ reduction of CD44 and CD133 in SW480/shR-OCT4B1. In addition, the protein level of CD44 and CD133 was reduced in the drug-resistant cells of SW480/shR-OCT4B1-OXR (Fig. 3B). These results suggested that OCT4B1 enhances cell survival by accelerating cell cycle progression, reducing apoptosis and maintaining EC cell property in colon cancer.

Overexpression of $O C T 4 B 1$ reduces sensitivity to oxaliplatin in vitro. To investigate the role of OCT4B1 with regard to sensitivity to oxaliplatin, we confirmed cytotoxicity assays using OCT4B1-overexpressed stably expressed cells (SW480/OCT4B1 and SW620/OCT4B1). As shown in Fig. 4A, 
A
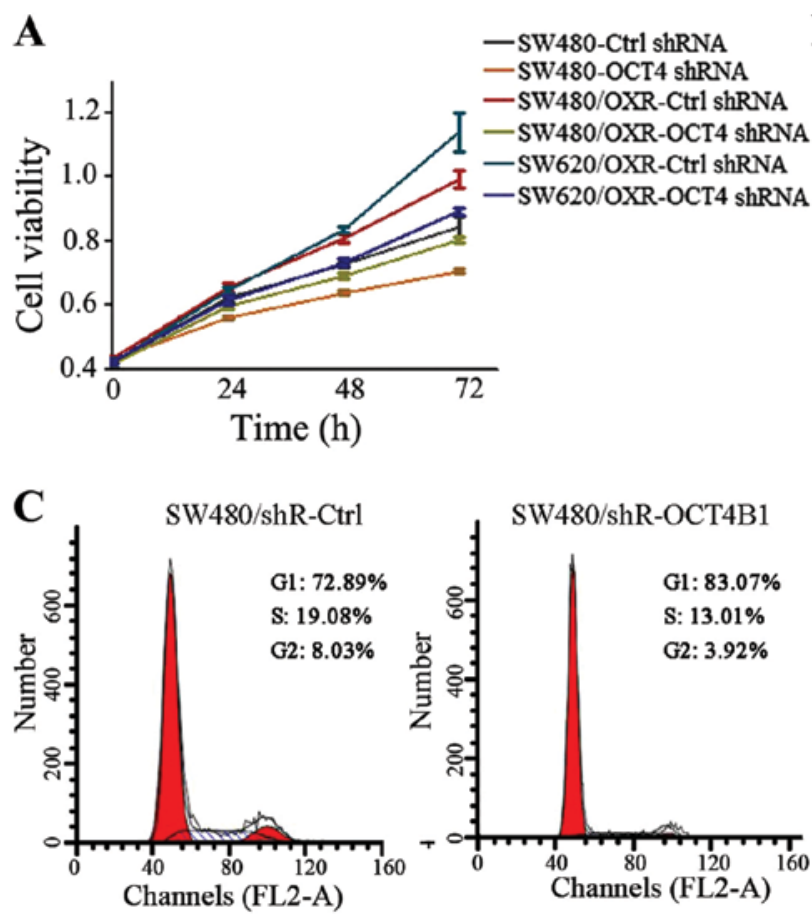

B

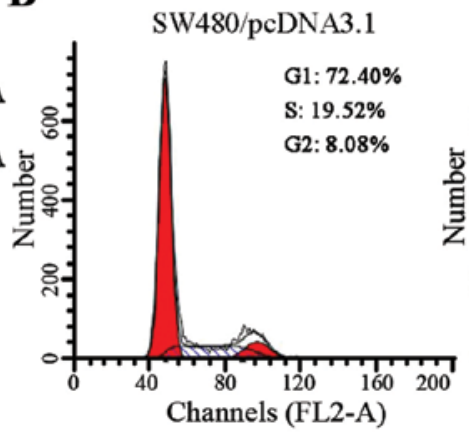

D

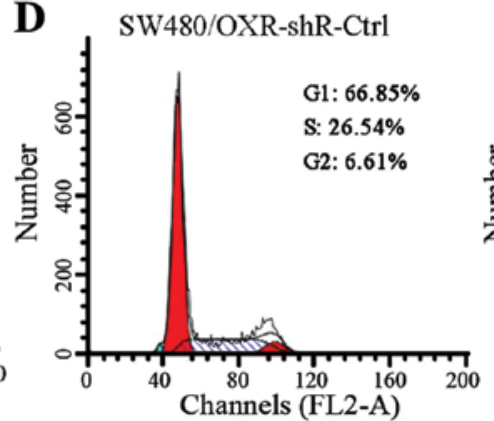

SW480/OCT4B1

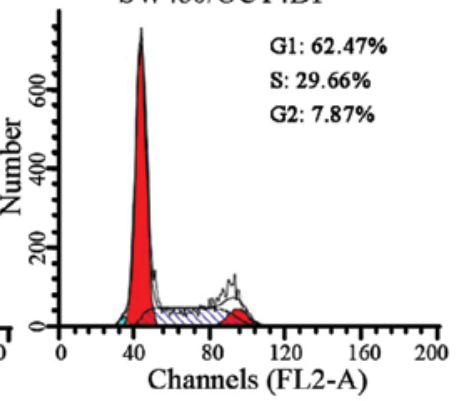

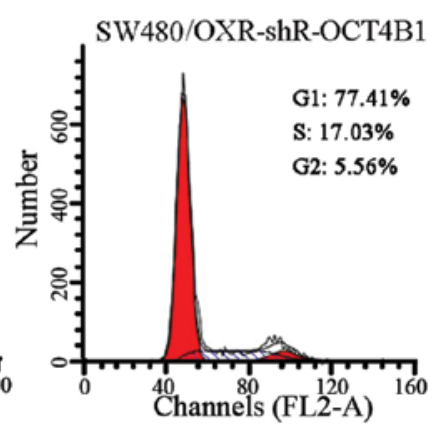

$\mathbf{E}$

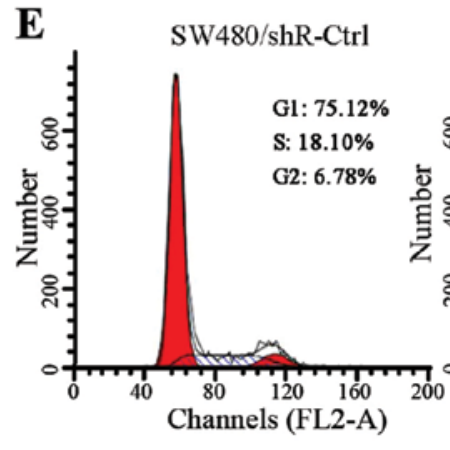

SW480/shR-OCT4B1

\section{$\mathbf{F}$}

SW480/OXR-shR-Ctrl
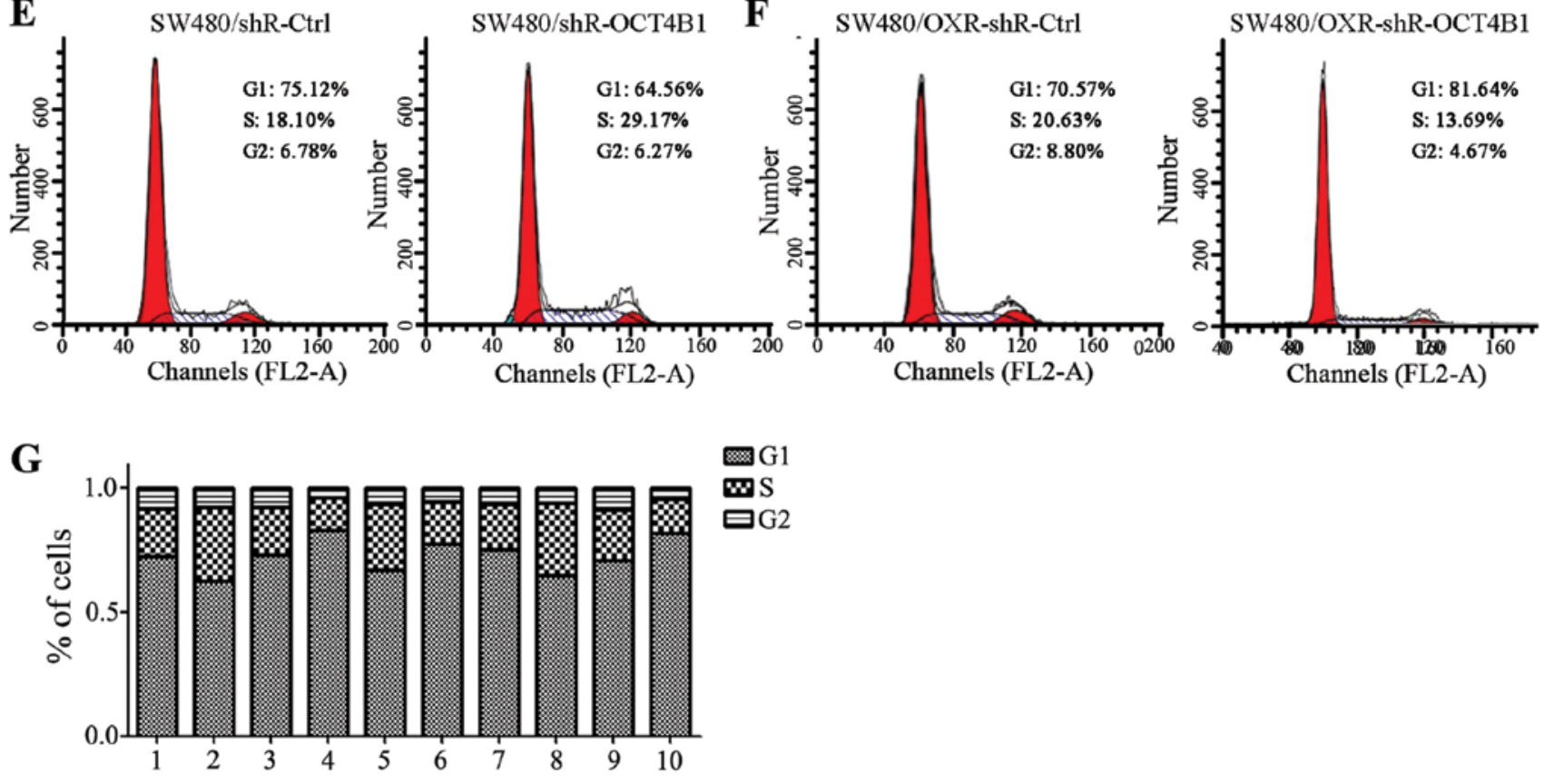

Figure 2. OCT4B1 affects cell viability and cell cycle in SW480 and SW620 cells in vitro. (A) The effect of OCT4B1 on cell viability was assessed by MTT assays at 24, 48 and $72 \mathrm{~h}$. (B-F) Distribution and the proportion of cells in three phases (G1, S, and G2) of the cell cycle in SW480 and SW620 cells as determined by FCM analysis. (G) The percentage of cells in each phase. OCT4, octamer-binding protein 4; FCM, flow cytometry.

the viability of SW480/OCT4B1 was significantly higher than that of the negative control. The amount of oxaliplatin requirement was much higher in SW480/OCT4B1 to achieve the same level of cell death as the SW480/pcDNA3.1. The respective $\mathrm{IC}_{50}$ doses for oxaliplatin were $2.5 \mathrm{~g} / \mathrm{ml}$ (SW480/OCT4B1) and $1.25 \mathrm{~g} / \mathrm{ml}$ (SW480/pcDNA3.1). Similar results were obtained in the SW620/OCT4B1 and SW620/pcDNA3.1 groups (Fig. 4A).

As P-gp and ABCG2 play important roles on the drug resistance of cancer cells, we examined the mechanism of reduced sensitivity to oxaliplatin by OCT4B1. Overexpression of OCT4B1 led to an increase while the knockdown of OCT4B1 led to a decrease in the level of P-gp and ABCG2 (Fig. 4B and C). In the drug-resistant cells, knockdown of OCT4B1 suppressed the expression of P-gp and ABCG2 in SW480/OXR-shR-OCT4B1 and SW620/OXR-shR-OCT4B1 compared to the control group (Fig. 4B and C). These results suggested that OCT4B1 reduces sensitivity to oxaliplatin by increasing the two important mediators of the drug resistance process in colon cancer.

OCT4B1 promotes migration and invasion via regulation of the EMT process in vitro. Migration and invasion are essential for tumor metastasis. To determine whether 


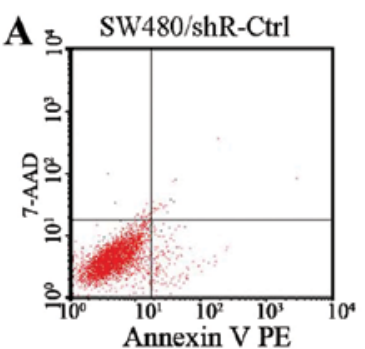

SW480/shR-OCT4B1
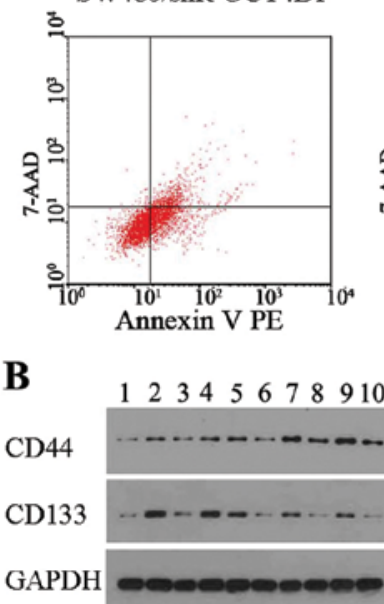

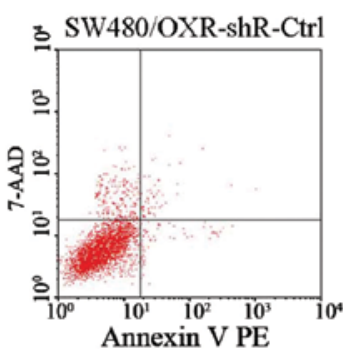

SW480/OXR-shR-OCT4B1
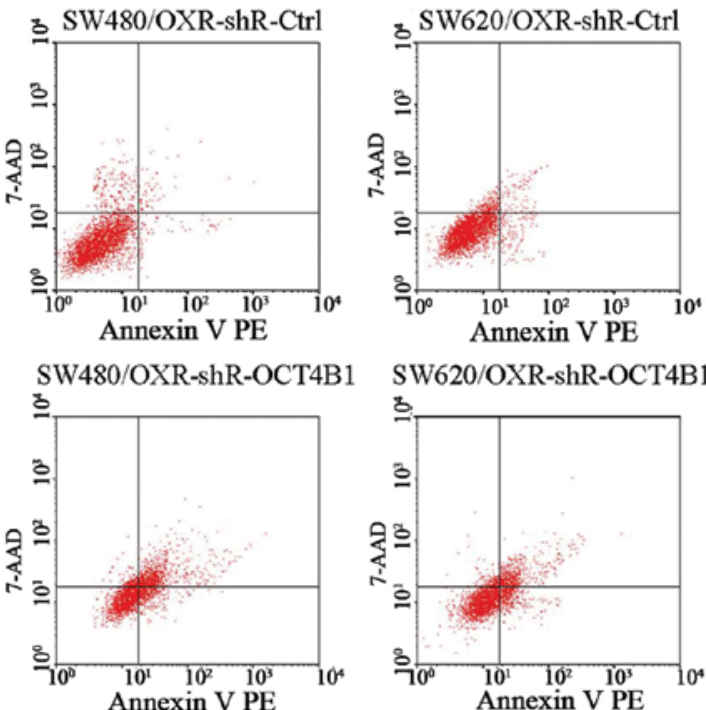

SW620/OXR-shR-OCT4B1
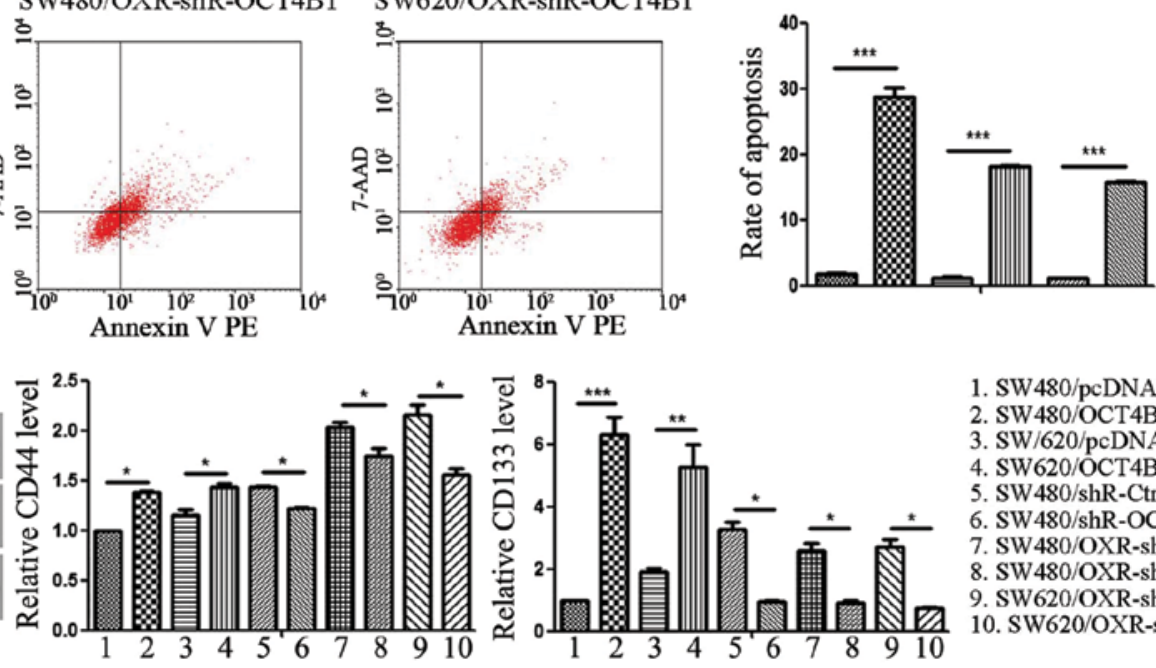

SW480/pcDNA3.1 2. SW480/OCT4B1 3. SW/620/pcDNA3,1 4. SW620/OCT4B1 5. SW480/shR-Ctrl 6. SW480/shR-OCT4B1 7. SW480/OXR-shR-Ctr 8. SW480/OXR-shR-OCT4B1 9. SW620/OXR-shR-Ctrl 10. SW620/OXR-shR-OCT4B1

Figure 3. OCT4B1 affects cell viability in SW480 and SW620 cells in vitro. (A) FCM was used to determine the apoptosis index of SW480 and SW620 cells. (B) The expression of CD44 and CD133 in the stably expressed cells. GAPDH was used as the control. OCT4, octamer-binding protein $4 .{ }^{*} \mathrm{P}<0.05$, ${ }^{* * *} \mathrm{P}<0.01$. and ${ }^{* * *} \mathrm{P}<0.001$

B

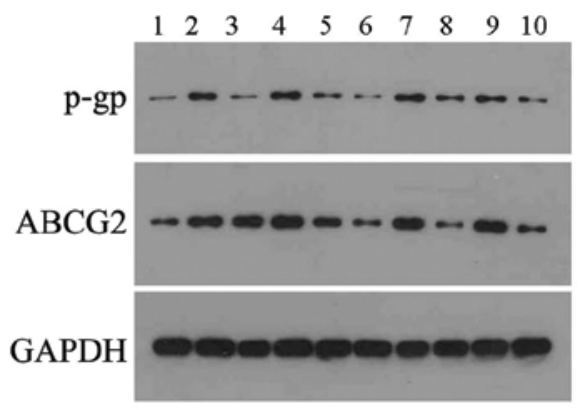

A

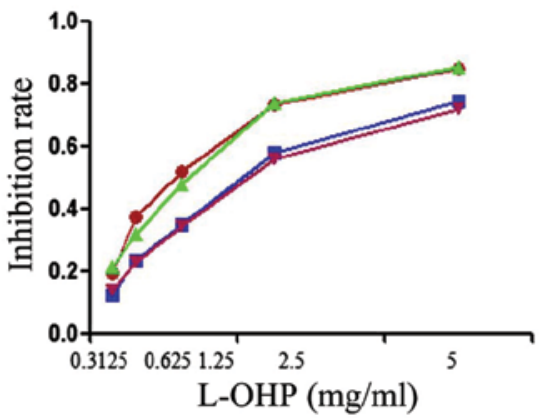

C
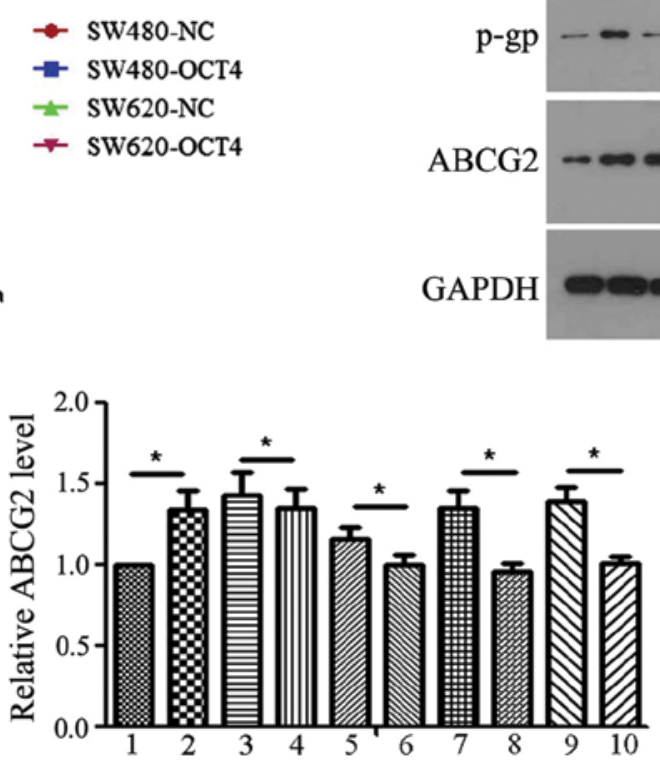

1. SW480/pcDNA3.1 2. SW480/OCT4B1 3. SW/620/pcDNA3,1 4. SW620/OCT4B1

5. SW480/shR-Ctrl

6. SW480/shR-OCT4B1

7. SW480/OXR-shR-Ctrl 8. SW480/OXR-shR-OCT4B1 9. SW620/OXR-shR-Ctrl

10. SW620/OXR-shR-OCT4B1

Figure 4. OCT4B1 reduces sensitivity to oxaliplatin in vitro. (A) SW480/OCT4B1 and SW620/OCT4B1 were seeded in 96-well plates. A cell cytotoxicity assay was performed at $24 \mathrm{~h}$ assessed by MTT assays after the cells were seeded. (B) The expression of P-gp and ABCG2 in the stably expressed cells. GAPDH was used as the control. (C) The quantification of expression of P-gp and ABCG2 in western blot analysis. OCT4, octamer-binding protein 4; P-gp, P-glycoprotein. $\mathrm{P}<0.05$ and ${ }^{* *} \mathrm{P}<0.01$

OCT4B1 can affect the migration and invasion properties of colon cancer cells, wound-scratch and Transwell assays with Matrigel were carried out in the stably expressed cells.
The wound-scratch assay showed that overexpression of OCT4B1 promoted wound recovery in SW480/OCT4B1 and SW620/OCT4B1 cells. By contrast, the inhibition of 

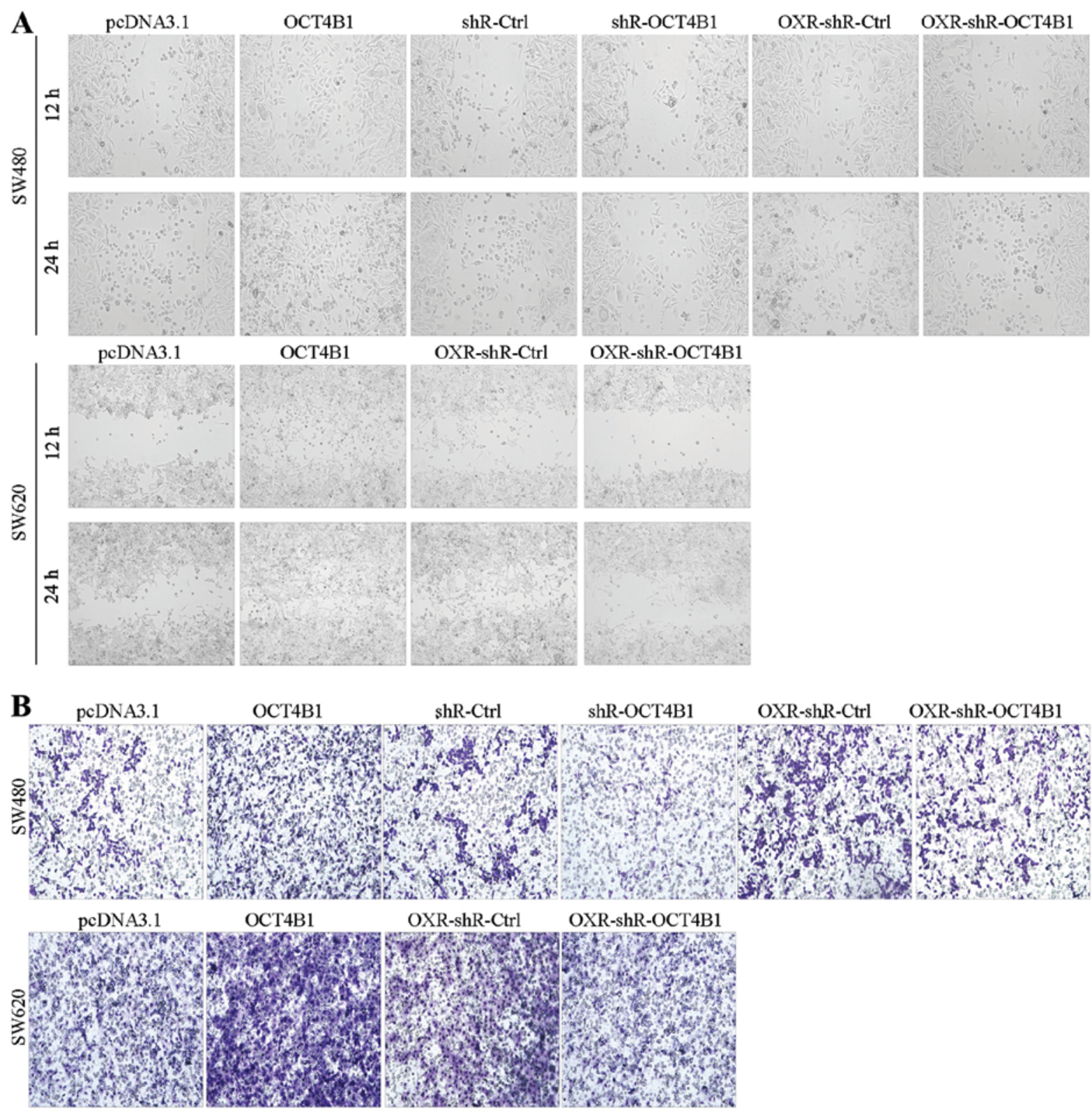

C

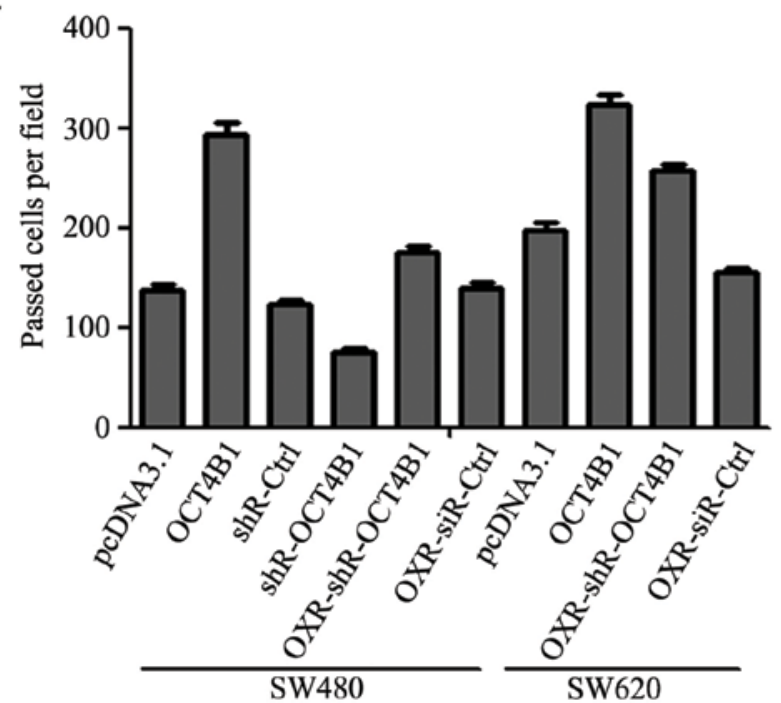

Figure 5. OCT4B1 promotes cell migration and invasion of colon cancer cells in vitro. (A) In vitro wound-healing assay was carried out with SW480 or SW620 cells or drug-resistant cells after being seeded at 12 and $24 \mathrm{~h}$. (B) Transwell invasion assays with Matrigel were used to detect the invasion activity of OCT4B1 in SW480 or SW620 cells or drug-resistant cells. (C) The quantification of three randomly selected fields shows the passed cells in Transwell invasion assays. OCT4, octamer-binding protein 4. 


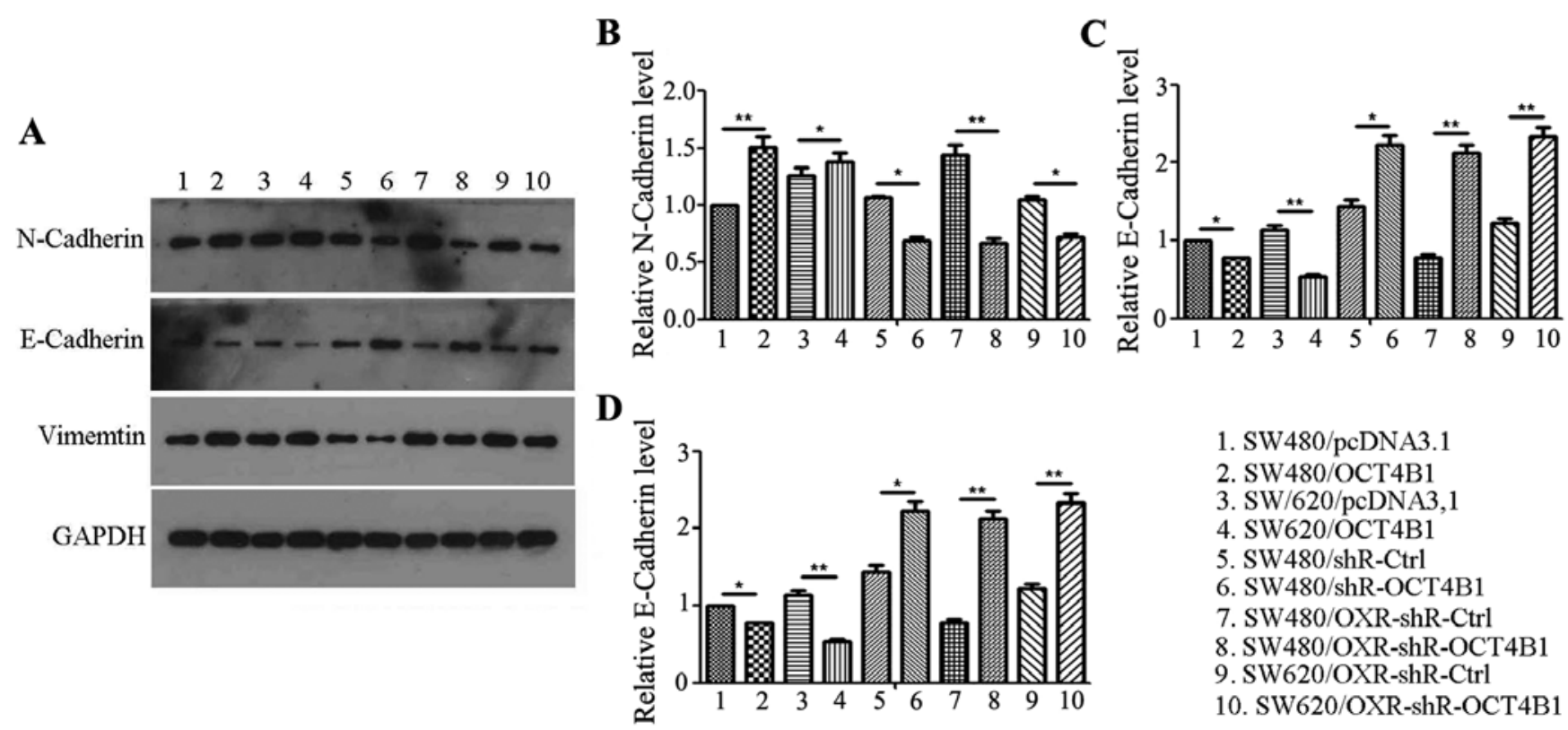

Figure 6. OCT4B1 promotes cell migration and invasion via EMT alteration. (A) The expression of N-cadherin, E-cadherin and vimentin in the stably expressed cells. GAPDH was used as the control. (B-D) The quantification of the expression of N-cadherin, E-cadherin and vimentin in western blot analysis. OCT4, octamer-binding protein 4; EMT, epithelial-to-mesenchymal transition. $\mathrm{P}<0.05$ and ${ }^{* *} \mathrm{P}<0.01$.

OCT4B1 delayed wound recovery in SW480/shR-OCT4B1 compared with the control group (Fig. 5A). We also examined the effects of OCT4B1 on drug-resistant cells. As expected, knockdown of OCT4B1 delayed wound recovery in drug resistance cells (Fig. 5A). The drug-resistant cells, SW480/OXR-shR-OCT4B1 and SW620/OXR-shR-OCT4B1, obtained the same results (Fig. 5A). Transwell assay with Matrigel showed that overexpression of OCT4B1 increased cell invasion 2.12 - and 1.64-fold in SW480 and SW620 cells overexpressed with OCT4B1, respectively, compared with the control group (Fig. 5B and C). The inhibition of OCT4B1 resulted in a 39.40, 20.11 and $40.05 \%$ reduction in SW480/shR-OCT4B1, SW480/OXR-shR-OCT4B1 and SW620/OXR-shR-OCT4B1, respectively (Fig. 5B and C). These results indicated that OCT4B1 promoted the migration and invasion of colon cancer in vitro.

As the results showed that OCT4B1 promotes the migration and invasion of colon cancer, we investigated the mechanism underlying the function of OCT4B1 in colon cancer. First, we examined the typical molecular alterations of EMT. The expression of the adherent marker E-cadherin and tight junction marker $\mathrm{N}$-cadherin and vimentin was examined. The protein level of E-cadherin was reduced $23.01 \%$ in SW480/OCT4B1 single-cell cloning cells compared with the negative control (Fig. 5A and C). The level of N-Cadherin and vimentin was increased 50.21 and $25.52 \%$, respectively, in SW480/OCT4B1 stably expressed cells (Fig. 6A, B and D). In the SW480/shR-OCT4B1 group, the protein level of E-cadherin was increased 55.23\%, whereas $\mathrm{N}$-cadherin and vimentin was reduced 53.96 and $8.21 \%$, respectively, compared with the control group (Fig. 6). The alteration of EMT was also examined in the drug-resistant cells. The expression of E-cadherin increased whereas that of $\mathrm{N}$-cadherin and vimentin was reduced in SW480/OXR-shR-OCT4B1 and
SW620/OXR-shR-OCT4B1 cells compared with the control group, suggesting that OCT4B1 participated in the malignant phenotype of drug resistance in colon cancer (Fig. 6). Similar results were obtained in SW620 cells (Fig. 6). These results indicated that OCT4B1 promotes the migration and invasion of colon cancer through regulation of the EMT process.

\section{Discussion}

OCT4B1, also known as POU5F1, is a novel transcript in the human OCT4 gene, which appears to be a master regulator in maintaining the pluripotency and self-renewal of ES cells (7). Since OCT4A and OCT4B were identified in 1992, studies have focused on the activity of the transcription factor responsible for the stemness properties of OCT4A and the cell stress-related funtion of OCT4B $(20,21)$. The function of OCT4B1 in pluripotency and self-renewal has been identified. Asadzadeh et al have reported that OCT4B1 is expressed in pleuripotent cells (15). Based on the characteristic of stem cells, the cancer stem cell (CSC) renders the self-renewal potential, tumorigenesis, and resistance to therapy characteristic of cancer (22). The high tumorigenicity of cancer cells occurred as a result of the dysregulation of the self-renewal process in stem cells $(23,24)$. It was previously reported that OCT4 variants may function as oncogenes or tumor-suppressing genes in cancer. In gastric adenocarcinoma, OCT4B1 acts as an anti-apoptotic factor (25). Addtionally, OCT4B1 has been expressed in gastric and colorectal cancers $(25,26)$. However, the role of OCT4B1 in the development of colon cancer remains to be determined. The present study focused on the contribution of OCT4B1 in the pathogenesis of colon cancer as well as cancer drug-resistant cells.

Identification of the role of OCT4B1 is critical for understanding the molecular mechanisms in tumorigenesis. We 
first screened the stably expressed cells overexpressing or knocking down OCT4B1 in SW480 and SW620 cells and their drug-resistant cells, respectively. Subsequently, we examined the effect of OCT4B1 on cell growth. In the present study, we found that OCT4B1 promotes cell growth in colon cancer and drug-resistant cells, which indicates that OCT4B1 participates in the growth of colon cancer as well as drug-resistant cells. Furthermore, we examined the mechanisms of the growth supporting role of OCT4B1 in colon cancer. First, we found that OCT4B1 promotes the ES cell property. Cell cycle and apoptosis were then detected in the OCT4B1-altered cells. The cell cycle analysis indicated that OCT4B1 preferentially promotes cell proliferation by facilitating the G1/S and S/G2 phase transitions, while OCT4B1 reduced cell apoptosis in colon cancer and drug-resistant cells. Thus, OCT4B1 promotes the growth of cancer cells via the ES cell property as well as alterations in the cell cycle and apoptosis.

In addition, we found that OCT4B1 reduced sensitivity to oxaliplatin, which further supports its oncogenic role in colon cancer. It is well known that the ABC transporter family participates in the formation of cancer drug resistance (27). P-gp and ABCG2 are two major mediators of drug resistance in cancer (28). Therefore, we hypothesized that OCT4B1 reduced the sensitivity to oxaliplatin due to the alteration of ABC transporter family expression. As expected, OCT4B1 was able to increase the expression of P-gp and ABCG2, which explains that OCT4B1 reduced the sensitivity to oxaliplatin in colon cancer. These results suggest that OCT4B1 reduces the sensitivity to oxaliplatin and potentiates $\mathrm{P}$-gp and ABCG2-mediated drug resistance.

Another important characteristic of cancer is metastasis. This is a complicated event whereby cells detach from the primary tumors, increase motility and invade the tissue stroma. The invasive tumor cells enter the circulation through blood vessels or lymphatic channels (29). Subsequently, the circulating tumor cells invade another organ and undergo metastatic growth (30). During this process, the cancer cells undergo an epithelial-mesenchymal transition (EMT) transition. Thus, we determined the effect of OCT4B1 on migration and invasion in colon cancer. The wound-healing assay revealed that OCT4B1 promotes the migarion of colon cancer and drug-resistant cells. Similarly, OCT4B1 contributes to the invasion in colon cancer performed by invasion assays. Of note, the migratiory and invasive abilities of OCT4B1 are resulted from EMT (31). OCT4B1 promotes the switch from the biomarker of the epithelial cells, E-cadherin, to vimentin and $\mathrm{N}$-cadherin, which are the mesenchymal biomarkers. These results suggest that OCT4B1 enhances the EMT by a reduction in the levels E-cadherin and an increase in the level of vimentin and N-cadherin, further stimulating the ability of migration and invasion in the colon cancer and drug-resistant cells.

In summary, results of the present study have demonstrated a novel function by which OCT4B1 stimulated colon cancer cell growth, migration, invasion and suppression of the sensitivity to oxaliplatin. In addition, OCT4B1 contributes the malignant phenotype of drug resistance in colon cancer cells. Therefore, inhibition of OCT4B1 may be considered to be a novel molecular treatment strategy for colon cancer and the drug resistance patients in the future.

\section{Acknowledgements}

This study was supported by the National Natural Science Foundation of China (no. 81260369) and the Science and Technology Fund Foundation of Guizhou [no. (2012)2365].

\section{References}

1. Slattery ML and Fitzpatrick FA: Convergence of hormones, inflammation, and energy-related factors: A novel pathway of cancer etiology. Cancer Prev Res (Phila) 2: 922-930, 2009.

2. Jemal A, Siegel R, Xu J and Ward E: Cancer statistics, 2010. CA Cancer J Clin 60: 277-300, 2010.

3. Grady WM and Carethers JM: Genomic and epigenetic instability in colorectal cancer pathogenesis. Gastroenterology 135: 1079-1099, 2008

4. Vogelstein B and Kinzler KW: Cancer genes and the pathways they control. Nat Med 10: 789-799, 2004.

5. Hofmanová J, Straková N, Vaculová AH, Tylichová Z, Safaříková B, Skender B and Kozubík A: Interaction of dietary fatty acids with tumour necrosis factor family cytokines during colon inflammation and cancer. Mediators Inflamm 2014: 848632, 2014.

6. Chen D, Yu Z, Zhu Z and Lopez CD: The p53 pathway promotes efficient mitochondrial DNA base excision repair in colorectal cancer cells. Cancer Res 66: 3485-3494, 2006.

7. Nichols J,Zevnik B, Anastassiadis K, Niwa H, Klewe-Nebenius D, Chambers I, Schöler H and Smith A: Formation of pluripotent stem cells in the mammalian embryo depends on the POU transcription factor Oct4. Cell 95: 379-391, 1998.

8. Fankam AG, Kuiate JR and Kuete V: Antibacterial activities of Beilschmiedia obscura and six other Cameroonian medicinal plants against multi-drug resistant Gram-negative phenotypes. BMC Complement Altern Med 14: 241, 2014.

9. Sui H, Liu X, Jin BH, Pan SF, Zhou LH, Yu NA, Wu J, Cai JF, Fan ZZ, Zhu HR, et al: Zuo Jin Wan, a traditional Chinese herbal formula, reverses P-gp-mediated MDR in vitro and in vivo. Evid Based Complement Alternat Med 2013: 957078, 2013.

10. Sui H, Fan ZZ and Li Q: Signal transduction pathways and transcriptional mechanisms of ABCB1/Pgp-mediated multiple drug resistance in human cancer cells. J Int Med Res 40: 426-435, 2012.

11. Atlasi Y, Mowla SJ, Ziaee SA, Gokhale PJ and Andrews PW: OCT4 spliced variants are differentially expressed in human pluripotent and nonpluripotent cells. Stem Cells 26: 3068-3074, 2008.

12. Wang X and Dai J: Concise review: Isoforms of OCT4 contribute to the confusing diversity in stem cell biology. Stem Cells 28: $885-893,2010$

13. Li R, Liang J, Ni S, Zhou T, Qing X, Li H, He W, Chen J, Li F, Zhuang Q, et al: A mesenchymal-to-epithelial transition initiates and is required for the nuclear reprogramming of mouse fibroblasts. Cell Stem Cell 7: 51-63, 2010.

14. Li Z, Li X, Li C, Su Y, Fang W, Zhong C, Ji W, Zhang Q and $\mathrm{Su} C$ : Transcription factor OCT4 promotes cell cycle progression by regulating CCND1 expression in esophageal carcinoma. Cancer Lett 354: 77-86, 2014

15. Asadzadeh J, Asadi MH, Shakhssalim N, Rafiee MR, Kalhor HR, Tavallaei M and Mowla SJ: A plausible anti-apoptotic role of up-regulated OCT4B1 in bladder tumors. Urol J 9: 574-580, 2012.

16. Asadi MH, Mowla SJ, Fathi F, Aleyasin A, Asadzadeh J and Atlasi Y: OCT4B1, a novel spliced variant of OCT4, is highly expressed in gastric cancer and acts as an antiapoptotic factor. Int J Cancer 128: 2645-2652, 2011.

17. Mirzaei MR, Najafi A, Arababadi MK, Asadi MH and Mowla SJ: Altered expression of apoptotic genes in response to OCT4B1 suppression in human tumor cell lines. Tumour Biol 35: 9999-10009, 2014.

18. Valianou M, Cox AM, Pichette B, Hartley S, Paladhi UR and Astrinidis A: Pharmacological inhibition of polo-like kinase 1 (PLK1) by BI-2536 decreases the viability and survival of hamartin and tuberin deficient cells via induction of apoptosis and attenuation of autophagy. Cell Cycle 14: 399-407, 2015.

19. Chen L, Zhang JJ and Huang XY: cAMP inhibits cell migration by interfering with Rac-induced lamellipodium formation. J Biol Chem 283: 13799-13805, 2008. 
20. Lee J, Kim HK, Rho JY, Han YM and Kim J: The human OCT-4 isoforms differ in their ability to confer self-renewal. J Bio Chem 281: 33554-33565, 2006.

21. Wang X, Zhao Y, Xiao Z, Chen B, Wei Z, Wang B, Zhang J, Han J, Gao Y, Li L, et al: Alternative translation of OCT4 by an internal ribosome entry site and its novel function in stress response. Stem Cells 27: 1265-1275, 2009.

22. Pardal R, Clarke MF and Morrison SJ: Applying the principles of stem-cell biology to cancer. Nat Rev Cancer 3: 895-902, 2003

23. Al-Hajj M and Clarke MF: Self-renewal and solid tumor stem cells. Oncogene 23: 7274-7282, 2004.

24. Lobo NA, Shimono Y, Qian D and Clarke MF: The biology of cancer stem cells. Annu Rev Cell Dev Biol 23: 675-699, 2007.

25. Junker K, Wolf M and Schubert J: Molecular clonal analysis of recurrent bladder cancer. Oncol Rep 14: 319-323, 2005.

26. Engers R: Reproducibility and reliability of tumor grading in urological neoplasms. World J Urol 25: 595-605, 2007.
27. Wang XK, To KK, Huang LY, Xu JH, Yang K, Wang F, Huang ZC, Ye $S$ and Fu LW: Afatinib circumvents multidrug resistance via dually inhibiting ATP binding cassette subfamily G member 2 in vitro and in vivo. Oncotarget 5: 11971-11985, 2014.

28. Greenberg RM: Schistosome ABC multidrug transporters: From pharmacology to physiology. Int J Parasitol Drugs Drug Resist 4: 301-309, 2014.

29. Chambers AF, Groom AC and MacDonald IC: Dissemination and growth of cancer cells in metastatic sites. Nat Rev Cancer 2: 563-572, 2002

30. Servant G, Weiner OD, Herzmark P, Balla T, Sedat JW and Bourne HR: Polarization of chemoattractant receptor signaling during neutrophil chemotaxis. Science 287: 1037-1040, 2000.

31. Thiery JP: Epithelial-mesenchymal transitions in tumour progression. Nat Rev Cancer 2: 442-454, 2002. 